HU-EP-12/49

\title{
Conformal geometry of null hexagons for Wilson loops and scattering amplitudes
}

\author{
Harald Dorn 1, Hagen Münkler and Christian Spielvogel \\ Institut für Physik der Humboldt-Universität zu Berlin, \\ Newtonstraße 15, D-12489 Berlin, Germany
}

\begin{abstract}
The cross-ratios do not uniquely fix the class of conformally equivalent configurations of null polygons. In view of applications to Wilson loops and scattering amplitudes we characterise all conformal classes of null hexagon configurations belonging to given points in cross-ratio space. At first this is done for the ordered set of vertices. Including the edges, we then investigate the equivalence classes under conformal transformations for null hexagons. This is done both for the set of null hexagons closed in finite domains of Minkowski space as well as for the set including those closed via infinity.
\end{abstract}

\footnotetext{
${ }^{1}$ dorn@physik.hu-berlin.de
} 


\section{Introduction}

Wilson loops as non-local gauge invariant, but path dependent, quantities play a central role in any gauge field theory. A lot of studies in the past four decades were devoted to properties for generic contours, both without and with cusps or intersections. Often one is also free to switch to Euclidean space. However, in particular due to their relation to the infrared behaviour of QCD (see e.g. [1]), also the investigation of properties characteristic for Wilson loops in Minkowski space with its indefinite metric has a long history.

This aspect has gained further momentum by the discovery of the correspondence between Wilson loops for null-polygonal contours and scattering amplitudes in $\mathcal{N}=4$ super Yang-Mills gauge theory, first via the AdS-CFT correspondence for strong coupling [2] and soon afterwards also at weak coupling [3,4]. Null-polygonal contours are very special, insofar as they have no counterpart in Euclidean space. Furthermore, the dependence on the contour reduces to the dependence on the location of the vertices $\left\{x_{j}\right\}$ of the polygon and then by Poincaré invariance to the dependence on the related Mandelstam-like variables.

Conformal invariance, present on the classical level, is broken due to the presence of ultraviolet divergences. The behaviour of the dimensional regularised Wilson loops for null polygons is somehow controlled by anomalous conformal Ward identities [5]. It results in the so-called BDS structure plus, starting with hexagons, a remainder function which is conformally invariant. The BDS structure had been invented before for infrared regularised scattering amplitudes [6].

Counting the number of parameters of the conformal group, one finds for a null hexagon in $\mathbb{R}^{1,3}$ three remaining continuous parameters specifying classes of conformally equivalent configurations. The standard choice are the three cross-ratios

$$
u_{1}=\frac{x_{13}^{2} x_{46}^{2}}{x_{14}^{2} x_{36}^{2}}, \quad u_{2}=\frac{x_{24}^{2} x_{15}^{2}}{x_{25}^{2} x_{14}^{2}}, \quad u_{3}=\frac{x_{35}^{2} x_{26}^{2}}{x_{36}^{2} x_{25}^{2}},
$$

formed out of the Mandelstam variables

$$
x_{i j}^{2}:=\left(x_{i}-x_{j}\right)^{2} .
$$

Since the anomalous conformal Ward identities encode the consequences of the invariance of the remainder functions only with respect to infinitesimal conformal transformation it remains open, whether the invariance holds also for all finite transformations. Some early tests with special examples of different hexagon configurations having the same cross-ratios indicated agreement [4,7]. However, beyond some doubt concerning the conformal invariance of the remainder function, there is another reason for asking whether the cross-ratios fix its value. It is the purely geometrical question, whether the three cross-ratios fix the hexagon configuration uniquely, up to conformal maps. As pointed out in a footnote of [8], the answer is negative. This fact becomes more explicit by an example of two hexagon configurations with the same cross-ratios, but one with and the other without a pair of crossing edges [9]. Furthermore, there

it has also been shown, that the two loop remainder takes different values in both cases, but they are related by a suitable analytic continuation. 
The aim of the present paper is a complete characterisation, both of all triples of cross-ratios as well as of all different classes of conformally equivalent real configurations in $\mathbb{R}^{1,3}$, which belong to a given allowed point in the cross-ratio space. Special conformal transformations have a critical light cone which is mapped to infinity. If this cone cuts a certain edge of the hexagon, the image of this edge connects the two images of the adjacent vertices via infinity. Therefore, it is appropriate to proceed in two steps. At first to talk only about the identification of all the conformal invariants for an ordered set of six points, whose neighbours are light-like separated. This will be done in section 2. Including in a second step the edges, one has two options. If one wants to keep all conformal transformations in the game, one has to enlarge the set of null hexagons closed within Minkowski space by those who are closed only via infinity. On the other side, it seems to us also a legitimate question to ask for conformal classes of null hexagons, closed exclusively in a finite part of $\mathbb{R}^{1,3}$. Then one has to disregard special conformal transformations, whose critical light cone cuts the hexagon. In two parts of section 3 we explore these two different points of view.

In section 4 we add some comments on the location of some special hexagon configurations in the cross-ratio space. After a conclusion section a few technical points are collected in five appendices.

A last comment in this section concerns the nomenclature we are following below. The full set of conformal transformations is given by the group $O(2,4) / Z_{2}$, with $Z_{2}$ representing $\{1,-1\}$. However, if we talk about the conformal group and related conformal classes, we have in mind the component connected to the identity, i.e. $S O_{+}(2,4) / Z_{2} \cdot 2$

\section{Conformal classification of 6-tupels with light- like separated neighbours}

Minkowski space $\mathbb{R}^{1,3}$ can be mapped conformally and one to one to the cone [10]

$$
W_{0}^{2}+W_{0^{\prime}}^{2}-W_{1}^{2}-W_{2}^{2}-W_{3}^{2}-W_{4}^{2}=0
$$

in projective $\mathbb{R} P_{2,4}^{5}$ (i.e. equivalence classes of points in $\mathbb{R}^{2,4} \backslash\{0\}$ with $W \sim V \Leftrightarrow \Leftrightarrow$ $W=\lambda V, \quad \lambda \in \mathbb{R} \backslash\{0\})$. 3 The map is $\left(x \in \mathbb{R}^{1,3}\right)$

$$
\begin{aligned}
W^{\mu} & =\lambda x^{\mu}, \quad \mu=0,1,2,3, \\
W^{0^{\prime}} & =\frac{1}{2} \lambda\left(1-x^{\mu} x_{\mu}\right), \quad W^{4}=\frac{1}{2} \lambda\left(1+x^{\mu} x_{\mu}\right)
\end{aligned}
$$

and its inverse

$$
x^{\mu}=\frac{W^{\mu}}{W^{0^{\prime}}+W^{4}}
$$

\footnotetext{
${ }^{2}$ The other three components are generated by including time reversal $T$ and parity transformation $P$.

${ }^{3}$ We have added a subscript to $\mathbb{R} P^{5}$ to emphasise the role of the $(2,4)$ metric in the embedding space.
} 
For several uses of this formalism see also e.g. [11, [12, [13. Conformal infinity of Minkowski space is mapped to points $\lambda W, \quad W^{0^{\prime}}+W^{4}=0$.

For two arbitrary points $x_{i}, x_{j}$ one gets

$$
\left(x_{i}-x_{j}\right)^{2}=\frac{\left(W_{i}-W_{j}\right)^{2}}{\left(W_{i}^{0^{\prime}}+W_{i}^{4}\right)\left(W_{j}^{0^{\prime}}+W_{j}^{4}\right)}=\frac{-2 W_{i} W_{j}}{\left(W_{i}^{0^{\prime}}+W_{i}^{4}\right)\left(W_{j}^{0^{\prime}}+W_{j}^{4}\right)} .
$$

In particular, light-like separated points in $\mathbb{R}^{1,3}$ correspond to points on the cone (3), which are light-like separated in the sense of $\mathbb{R}^{2,4}$.

Remarkably, inserting ([6) into the cross ratios of type (11), the denominators of the r.h.s. cancel. Thus the cross-ratios can directly be expressed in terms of the distances of the related points on the cone in $\mathbb{R}^{2,4}$

$$
\frac{x_{i j}^{2} x_{k l}^{2}}{x_{i l}^{2} x_{k j}^{2}}=\frac{\left(W_{i} W_{j}\right)\left(W_{k} W_{l}\right)}{\left(W_{i} W_{l}\right)\left(W_{k} W_{j}\right)} .
$$

Each special conformal transformation

$$
x^{\prime \mu}=\frac{x^{\mu}+c^{\mu} x^{2}}{1+2 c x+c^{2} x^{2}}
$$

has a critical light cone in $\mathbb{R}^{1,3}$ with tip at $-\frac{c}{c^{2}}$. This whole light cone is mapped to conformal infinity. The point on $\mathbb{R} P_{2,4}^{5}$, corresponding to the image of the tip after applying (8), is found with appendix A, eq. (48)

$$
W_{\mathrm{tip}}^{N}=\lambda(0,0,0,0,-1,1) .
$$

Note that in this notation $N$ takes the values $0,1,2,3,0^{\prime}, 4$. The point $W_{\text {tip }}$ is invariant under dilatations, translations and Lorentz transformations of the original Minkowski space $\mathbb{R}^{1,3}$.

Let now $x_{1}$ to $x_{6}$ denote the vertices of our null hexagon and $W_{1}$ to $W_{6}$ their images on $\mathbb{R} P_{2,4}^{5}$. Then we apply a special conformal transformation, whose critical light cone has its tip at $x_{6}$, i.e. $c=-\frac{x_{6}}{x_{6}^{2}}$. This light cone $\left\{x_{6}+n \mid n^{2}=0\right\}$ is mapped to conformal infinity with the $\mathbb{R} P_{2,4}^{5}$ image

$$
\lambda\left(n^{\mu}-\frac{2 x_{6} n}{x_{6}^{2}} x_{6}^{\mu},-\frac{\left(x_{6}+n\right)^{2}}{2}, \frac{\left(x_{6}+n\right)^{2}}{2}\right) .
$$

By including an overall rescaling into the prefactor $\lambda$ we arrive at $\lambda\left(w^{\mu},-1,1\right)$ or $\lambda\left(w^{\mu}, 0,0\right)$. The last option corresponds to the exceptional points on the original light cone, which are also light-like with respect to the origin. We assume that $x_{1}$ and $x_{5}$ are not of exceptional type 4 .

After this we have

$$
W_{6}=\lambda_{6}(0,0,0,0,-1,1), \quad W_{1}=\lambda_{1}\left(w_{1}^{\mu},-1,1\right), \quad W_{5}=\lambda_{5}\left(w_{5}^{\mu},-1,1\right) .
$$

\footnotetext{
${ }^{4}$ In each neighbourhood of exceptional points one has non-exceptional ones. Hence for our purpose of determining the range of the three cross-ratios this is allowed.
} 
Starting from a generic configuration the remaining three points $x_{2}, x_{3}, x_{4}$ are then still finite in $\mathbb{R}^{1,3}$. By a translation we shift $x_{3}$ into the origin, which by (4) means

$$
W_{3}=\lambda_{3}(0,0,0,0,1,1) \text {. }
$$

Under translations in Minkowski space $x \mapsto x+a$ (Appendix A, (47)) $W=\lambda(w,-1,1)$ goes to $\lambda(w,-(1+a w), 1+a w)$. With the same justification, as given in the last footnote, we assume the non-exceptional case $1-x_{3} w_{j} \neq 0, j=1,5$, and find after rescaling, that this translation does not affect the structure of (10). Since $x_{2}$ and $x_{4}$ are light-like separated from $x_{3}=0$ the corresponding points on $\mathbb{R} P_{2,4}^{5}$ have now the form

$$
W_{2}=\lambda_{2}\left(w_{2}^{\mu}, 1,1\right), \quad W_{4}=\lambda_{4}\left(w_{4}^{\mu}, 1,1\right) .
$$

The cone condition (3) and the null condition for the hexagon edges via (6) constrain the entries $w_{1}^{\mu}, w_{2}^{\mu}, w_{4}^{\mu}$ and $w_{5}^{\mu}$ in (10) and (12) by

$$
w_{1} w_{2}=w_{4} w_{5}=2, \quad w_{j}^{2}=0, j=1,2,4,5 .
$$

At this stage we still have the freedom to use Lorentz transformations and dilatations to further specify the $w_{j}$. From Appendix A, (49) and (50) we see that the structure (10)-(12) is preserved and the entries $w_{j}, j=1,2,4,5$ are transformed like vectors in $\mathbb{R}^{1,3}$. For generic cases $\left(x_{2}-x_{4}\right)^{2} \neq 0$ one has $w_{2} w_{4} \neq 0$ and can use a dilatation to achieve $w_{2} w_{4}= \pm 2$. Let us first continue with

Case $A: w_{2} w_{4}=2$

Then by a suitable Lorentz transformation we get $w_{2}=( \pm|\vec{w}|, \vec{w}), \quad w_{4}=( \pm|\vec{w}|,-\vec{w})$. Rotating the Euclidean unit three vector $\vec{w}$ in the direction of the 1-axis we arrive at

$$
w_{2}=( \pm 1,1,0,0), \quad w_{4}=( \pm 1,-1,0,0) .
$$

Now all freedom to map a generic configuration via conformal transformations to a special subset of configurations has been used. $w_{1}$ and $w_{5}$ are constrained only by (13), which leads to the structure

$$
w_{1}=\left( \pm\left(1+\vec{p}^{2}\right), \vec{p}^{2}-1,2 \vec{p}\right), \quad w_{5}=\left( \pm\left(1+\vec{q}^{2}\right), 1-\vec{q}^{2}, 2 \vec{q}\right) .
$$

Here $\vec{p}$ and $\vec{q}$ are arbitrary two-dimensional Euclidean vectors.

Now using (10)-(12), (14), (15) and (7) for the evaluation of the cross-ratios (1) we get

$$
u_{1}=\frac{1}{1-\vec{p}^{2}}, \quad u_{2}=\frac{1+\vec{p}^{2} \vec{q}^{2}-2 \vec{p} \vec{q}}{\left(1-\vec{p}^{2}\right)\left(1-\vec{q}^{2}\right)}, \quad u_{3}=\frac{1}{1-\vec{q}^{2}}
$$

A similar analysis for

Case B: $w_{2} w_{4}=-2$

leads to

$$
\begin{aligned}
& w_{2}=( \pm 1,1,0,0), \quad w_{4}=(\mp 1,1,0,0), \\
& w_{1}=\left( \pm\left(1+\vec{p}^{2}\right), \vec{p}^{2}-1,2 \vec{p}\right), \quad w_{5}=\left(\mp\left(1+\vec{q}^{2}\right), \vec{q}^{2}-1,2 \vec{q}\right),
\end{aligned}
$$


as well as

$$
u_{1}=\frac{1}{1+\vec{p}^{2}}, \quad u_{2}=\frac{1+\vec{p}^{2} \vec{q}^{2}+2 \vec{p} \vec{q}}{\left(1+\vec{p}^{2}\right)\left(1+\vec{q}^{2}\right)}, \quad u_{3}=\frac{1}{1+\vec{q}^{2}} .
$$

The domain in the three-dimensional space of cross-ratios, which can be realised by real null hexagon configurations in Minkowski space, is fully covered by (16) and (18) varying independently the three variables $p, q, z\left(p=|\vec{p}|, q=|\vec{q}|, z=\frac{\vec{p} \vec{q}}{p q}\right)$ within $p, q \geq 0$ and $-1 \leq z \leq 1$. Expressing $p$ and $q$ in terms of $u_{1}$ and $u_{3}$ one finds ( $\mp$ refers to case $\mathrm{A} / \mathrm{B})$

$$
u_{2}=2 u_{1} u_{3}-u_{1}-u_{3}+1 \mp 2 z \sqrt{u_{1} u_{3}\left(1-u_{1}\right)\left(1-u_{3}\right)} .
$$

This domain is depicted in fig.1.

Its characterisation in terms of the $u_{j}$ alone is given by the overall inequality

$$
4 u_{1} u_{2} u_{3}-\left(u_{1}+u_{2}+u_{3}-1\right)^{2} \geq 0,
$$

valid in the whole domain. Its five parts, in addition, are specified by

$$
\begin{aligned}
\text { "bag" } & : \quad 0 \leq u_{k} \leq 1, \quad k=1,2,3, \\
\text { "ear j", } j=1,2,3: & u_{j} \geq 1, \quad u_{k} \leq 0, \quad k \neq j \\
\text { "ear 4" }: & u_{k} \geq 1, \quad k=1,2,3
\end{aligned}
$$

The central bag is realised with case B. The four ears are realised with case A.

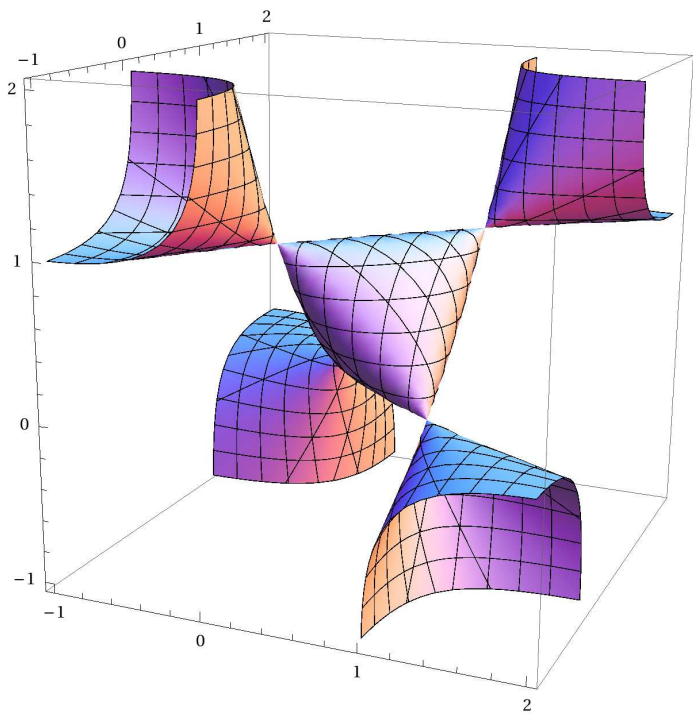

Figure 1: The allowed region for $\mathbb{R}^{1,3}$ null hexagons in cross-ratio space.

Note also that the two options, which one has both in case A and B by the choice of the upper/lower signs in (14), (15) and (17), are related by time reversal in Minkowski space, corresponding to $W^{0} \rightarrow-W^{0}$ in $\mathbb{R}^{2,4}$. 
In reconstructing the 2 -dimensional vectors $\vec{p}$ and $\vec{q}$ from the cross-ratios, i.e. from $\vec{p}^{2}, \vec{q}^{2}, \vec{p} \vec{q}$, see (16), (18), besides the freedom of rotations, one has the option $\vartheta \rightarrow-\vartheta$ for the angle between $\vec{p}$ and $\vec{q}$. Furthermore, in (14), (15) the three 3D vectors $\vec{w}, \vec{w}_{1}$ and $\vec{w}_{5}$ are involved. After we decided to rotate $\vec{w}$ in the direction of the 1-axis, the 3D orientation of this triple of vectors manifests itself in the $2 \mathrm{D}$ orientation of the pair $\vec{p}, \vec{q}$ parameterising $\vec{w}_{1}$ and $\vec{w}_{5}$. Hence a change of this orientation is due to a parity transformation in Minkowski space.

Finally, one should add the trivial observation, that under a cyclic shift of the numbering of the points $\left\{x_{j}\right\}$ one has

$$
\left(x_{1}, x_{2}, x_{3}, x_{4}, x_{5}, x_{6}\right) \mapsto\left(x_{6}, x_{1}, x_{2}, x_{3}, x_{4}, x_{5}\right) \Longrightarrow\left(u_{1}, u_{2}, u_{3}\right) \mapsto\left(u_{3}, u_{1}, u_{2}\right) .
$$

Such a shift maps both the bag and ear 4 to itself and implies a corresponding cyclic mapping of the ears 1 to 3 .

Altogether we have shown that each ordered set of six light-like separated points $\left(x_{1}, \ldots, x_{6}\right)$ in Minkowski space can be mapped by conformal transformations to one of the four standard configurations, whose image on $\mathbb{R} P_{2,4}^{5}$ has been described above. Since case A and B cover different regions in the space of cross-ratios, this implies that a given generic point $\left(u_{1}, u_{2}, u_{3}\right)$ in the allowed region (20)-(23) fixes the position of $\left(x_{1}, \ldots, x_{6}\right)$ uniquely, up to conformal transformations, up to the cyclic permutation $\left(x_{1}, x_{2}, \ldots, x_{6}\right) \rightarrow\left(x_{4}, x_{5}, \ldots, x_{3}\right)$ and up to time reversal and up to a parity transformation. 5

\section{Conformal classification of null hexagons}

\subsection{Null hexagons closed via infinity included}

Topologically the null geodesic, which is determined by a given pair of two consecutive points $x_{j}, x_{j+1}$ out of a 6 -tuple of light-like separated points, is a circle on conformally compactified Minkowski space, i.e. on $\mathbb{R} P_{2,4}^{5}$, see Appendix B. The two points $x_{j}, x_{j+1}$ divide it into two parts. One of them contains a point at conformal infinity.

Let us first take into consideration also null hexagons which close via infinity, i.e. allow for the edge connecting $x_{j}$ and $x_{j+1}$ either the direct connection or the connection via infinity. Although crossing infinity is not a conformally invariant characteristic, it is nevertheless possible to mark the two parts of such a null geodesic in an $\mathrm{SO}_{+}(2,4)$ invariant way. The notion of a time arrow on null geodesics is $\mathrm{SO}_{+}(2,4)$ invariant $\left(\frac{d x^{0}}{d s}>0 \Rightarrow \frac{d\left(x^{\prime}\right)^{0}}{d s}>0\right)$. Therefore, the two parts can be distinguished by the alternative of going from $x_{j}$ to $x_{j+1}$ with or opposite to the time arrow.

After this remark we get for each set of cross-ratios $4 \times 2^{6}=256$ conformally inequivalent classes of hexagon configurations. Here the first factor 4 corresponds to time reversal and parity and the factor $2^{6}$ to the choice of one of the two parts of the null geodesics for all 6 pairs of consecutive vertices of the null hexagon.

\footnotetext{
${ }^{5}$ At non-generic points the degeneracy may be higher, e.g. if all $u_{j}$ are equal, each cyclic permutation is included. At the vertex points of the bag cases A and B are both possible.
} 
Under special conformal transformations

$$
\left(x^{\prime}-y^{\prime}\right)^{2}=\frac{(x-y)^{2}}{\left(c^{2}\right)^{2}\left(x+c / c^{2}\right)^{2}\left(y+c / c^{2}\right)^{2}} .
$$

Therefore, $J_{1}$ and $J_{2}$, defined by

$$
J_{1}:=\operatorname{sign}\left(x_{13}^{2} x_{35}^{2} x_{51}^{2}\right), \quad J_{2}:=\operatorname{sign}\left(x_{24}^{2} x_{46}^{2} x_{62}^{2}\right)
$$

are conformal invariants. According to our result in the previous section they cannot contain information independent of the cross-ratios.

For later use it is instructive to see this more explicitly. At first from the definition of the cross-ratios in (1) one gets $J_{1} J_{2}=\operatorname{sign}\left(u_{1} u_{2} u_{3}\right)$.

Eqs.(21)-(23) imply $\operatorname{sign}\left(u_{1} u_{2} u_{3}\right) \geq 0$, which means for the generic situation of all

$x_{j, j+2}^{2} \neq 0$ (Here and below the indices are understood modulo 6 and the index of the cross-ratios modulo 3.)

$$
\prod_{j=1}^{6} x_{j, j+2}^{2}>0 \quad \text { and } \quad J_{1}=J_{2} .
$$

Finally, (6) allows to express $J_{1}$ and $J_{2}$ in terms of the $W_{j}$ (as for the cross-ratios the denominators on the r.h.s. of ([6]) turn out to be irrelevant)

$$
J_{1}=-\operatorname{sign}\left(\left(W_{1} W_{3}\right)\left(W_{3} W_{5}\right)\left(W_{5} W_{1}\right)\right)
$$

and similarly for $J_{2}$. Now the $J$ 's can be read off directly from the standard configurations in $\mathbb{R} P_{2,4}^{5}$, we find

$$
\begin{aligned}
& J_{1}=J_{2}=-1, \quad \text { case A, } \\
& J_{1}=J_{2}=+1, \quad \text { case B. }
\end{aligned}
$$

Since case A and B cover different regions of the cross-ratio space (ears versus bag), $J_{1}$ and $J_{2}$ yield no independent information on conformal equivalence classes (except at the four points where the bag and ears touch each other).

\subsection{Null hexagons closing via infinity excluded}

In the following we ask a modified question. We are now interested in the set of null hexagons which are closed without going via infinity. Then we have to restrict the allowed conformal transformations to those which do not map out of this subset. As a bonus, we now can use the invariance of the signs of all the Mandelstam variables to characterise different classes of conformally equivalent configurations belonging to a given allowed point in the cross-ratio space. This sign invariance follows from the observation, that a sign change of one of the $x_{i j}^{2}$ is necessarily connected with a map to a hexagon configuration passing conformal infinity. This is shown in appendix E.

Concerning special conformal transformations (25) implies that the distance between two not null separated points changes iff both points are on different sides of 
the critical light cone centred at $-c / c^{2}$. This means that special conformal transformations, whose critical light cone cuts any edge of the to be transformed hexagon, have to be excluded.

As explained in the introduction, via the correspondence between Wilson loops and gluon scattering amplitudes, our hexagons are also relevant for 6-point scattering amplitudes with momenta $\left\{p_{j} \mid j=1, \ldots, 6\right\}$ related to the edges of the hexagon via

$$
p_{j}:=x_{j+1}-x_{j}
$$

Since

$$
\begin{aligned}
& x_{j j+2}^{2}>0 \quad \Leftrightarrow \quad \operatorname{sign}\left(p_{j}^{0}\right)=\operatorname{sign}\left(p_{j+1}^{0}\right), \\
& x_{j j+2}^{2}<0 \quad \Leftrightarrow \quad \operatorname{sign}\left(p_{j}^{0}\right)=-\operatorname{sign}\left(p_{j+1}^{0}\right),
\end{aligned}
$$

one can relate the sequence of signs for all the Mandelstam variables $x_{j j+2}^{2}$ to the type of related scattering: $3 \rightarrow 3$ or $2 \leftrightarrow 4$ scattering. Then we find by explicit inspection of all possible cases, that all contributions to $3 \rightarrow 3$ yield $J_{1}=J_{2}=-1$ and all contributions to $2 \leftrightarrow 4$ scattering yield $J_{1}=J_{2}=+1$. Therefore, we can supplement (29) by

$$
\begin{aligned}
\text { ears } 1 \text { to } 4 & \Leftrightarrow \quad \text { case } A \quad \Leftrightarrow 3 \rightarrow 3 \text { scattering , } \\
\text { bag } & \Leftrightarrow \text { case } B \quad \Leftrightarrow 2 \leftrightarrow 4 \text { scattering . }
\end{aligned}
$$

Another useful fact is

$$
x_{j j+2}^{2}>0, x_{j+1 j+3}^{2}>0 \Rightarrow x_{j j+3}^{2}>0 .
$$

We now want to list all sign assignments (realisable by real configurations in Minkowski space) to the six Mandelstam variables of type $x_{j j+2}^{2}$ and the three of type $x_{j j+3}^{2}$. The choice for the first type fixes the correlation to the type of scattering. Therefore, we collect them together in sets with fixed signs for the $x_{j j+2}^{2}$. Due to the first condition in (27), for them only an even number of negative signs is allowed. In some cases (33) fixes already also the sign of some of the $x_{j j+3}^{2} .6$

Sets of sign assignments to the $x_{j j+2}^{2}$

\begin{tabular}{|l|l|l|c|c|}
\hline case & $x_{13}^{2}, \ldots, x_{62}^{2}$ & already fixed $x_{j j+3}^{2}$ & scattering & cycl. perm. \\
\hline \hline $\mathbf{a}$ & ------ & none & $3 \rightarrow 3$ & 1 \\
\hline $\mathbf{b}$ & ++---- & $x_{14}^{2}>0$ & $2 \leftrightarrow 4$ & 6 \\
\hline $\mathbf{c}$ & +-+--- & none & $3 \rightarrow 3$ & 6 \\
\hline $\mathbf{d}$ & +--+-- & none & $2 \leftrightarrow 4$ & 3 \\
\hline $\mathbf{e}$ & -+-+++ & $x_{14}^{2}, x_{52}^{2}>0$ & $2 \leftrightarrow 4$ & 6 \\
\hline $\mathbf{f}$ & -++-++ & $x_{52}^{2}>0$ & $3 \rightarrow 3$ & 3 \\
\hline
\end{tabular}

We have skipped cases $(++++++)$ forbidden by $\sum_{j} p_{j}=0$ and $(--++++)$,

\footnotetext{
${ }^{6}$ We discuss here the generic case, where all Mandelstam variables under discussion are different from zero, the degenerated cases will be commented below.
} 
which would correspond to an unphysical $1 \leftrightarrow 5$ transition. As long as we talk about a fixed numbering of the hexagon vertices, cases b), c) and e) each are one of 6 cyclic permutations of sign assignments (at fixed numbering of the vertices!). Cases d) and f) each are one of 3 cyclic permutations, case a) is unique in this respect. These numbers are indicated in the last column of the table.

Now we list for each case of this table the remaining possibilities for signs for $x_{14}^{2}, x_{25}^{2}, x_{36}^{2}$. This fixes then the signs of the cross-ratios, and we can locate the related position in cross-ratio space (bag, ears), see (21)-(23). If one of the crossratios is negative, the location is evident. In the case of all cross-ratios positive, the separation between bag and ear 4 can be made, since we already know, that the bag is possible only with $2 \leftrightarrow 4$ and ear 4 only with $3 \rightarrow 3$.

case a), sign assignments to the $x_{j j+3}^{2}$

\begin{tabular}{|c|c|c|}
\hline case & $x_{14}^{2}, x_{25}^{2}, x_{36}^{2}$ & location in $u$-space \\
\hline \hline $\mathbf{a} 1$ & +++ & ear 4 \\
\hline $\mathbf{a} 2$ & --- & ear 4 \\
\hline $\mathbf{a} 3$ & --+ & ear 2 \\
\hline $\mathbf{a} 4$ & -+- & ear 1 \\
\hline $\mathbf{a} 5$ & +-- & ear 3 \\
\hline $\mathbf{a} 6$ & ++- & ear 2 \\
\hline $\mathbf{a} 7$ & +-+ & ear 1 \\
\hline $\mathbf{a} 8$ & -++ & ear 3 \\
\hline
\end{tabular}

For case b) one of the $x_{j j+3}^{2}$ has already a fixed sign. Three of the four remaining options would yield cross-ratios located in ears 1 to 3 . Since this pattern has no realisation by real hexagon configurations, we are left with

case b), sign assignments to the $x_{j j+3}^{2}$

\begin{tabular}{|c|c|c|}
\hline case & $x_{14}^{2}, x_{25}^{2}, x_{36}^{2}$ & location in $u$-space \\
\hline \hline $\mathbf{b}$ & +-- & bag \\
\hline
\end{tabular}

In case c) one finds again 8 possibilities

case c), sign assignments to the $x_{j j+3}^{2}$

\begin{tabular}{|c|c|c|}
\hline case & $x_{14}^{2}, x_{25}^{2}, x_{36}^{2}$ & location in $u$-space \\
\hline \hline $\mathbf{c 1}$ & +++ & ear 2 \\
\hline $\mathbf{c 2}$ & --- & ear 2 \\
\hline $\mathbf{c 3}$ & --+ & ear 4 \\
\hline $\mathbf{c} 4$ & -+- & ear 3 \\
\hline $\mathbf{c 5}$ & +-- & ear 1 \\
\hline $\mathbf{c 6}$ & ++- & ear 4 \\
\hline $\mathbf{c 7}$ & +-+ & ear 3 \\
\hline $\mathbf{c} 8$ & -++ & ear 1 \\
\hline
\end{tabular}


In case d) six of the eight options for the $x_{j j+3}^{2}$ indicated in table (34) would give points in the ears, hence not realisable for a $2 \leftrightarrow 4$ configuration 7

case $\mathbf{d})$, sign assignments to the $x_{j j+3}^{2}$

\begin{tabular}{|c|c|c|}
\hline case & $x_{14}^{2}, x_{25}^{2}, x_{36}^{2}$ & location in $u$-space \\
\hline \hline $\mathbf{d}^{*}$ & +++ & bag \\
\hline $\mathbf{d}$ & --- & bag \\
\hline
\end{tabular}

Case e) appears in table (34) with two already fixed signs for the $x_{j j+3}^{2}$. Only one of the two remaining option gives points in the bag.

case e), sign assignments to the $x_{j j+3}^{2}$

\begin{tabular}{|c|c|c|}
\hline case & $x_{14}^{2}, x_{25}^{2}, x_{36}^{2}$ & location in $u$-space \\
\hline \hline $\mathbf{e}$ & ++- & bag \\
\hline
\end{tabular}

Finally, for case f) table (34) allows four options.

case f), sign assignments to the $x_{j j+3}^{2}$

\begin{tabular}{|c|c|c|}
\hline case & $x_{14}^{2}, x_{25}^{2}, x_{36}^{2}$ & location in $u$-space \\
\hline \hline $\mathbf{f 1}$ & +++ & ear 4 \\
\hline f2 & -+- & ear 1 \\
\hline f3 & -++ & ear 3 \\
\hline f4 & ++- & ear 2 \\
\hline \hline
\end{tabular}

So far we have used our previous results (32) and (33) to eliminate some sign options. It remains to decide, whether there exist further restrictions or whether all options listed in tables (35)-(40) indeed can be realised by real configurations in Minkowski space. If a certain sign option is allowed, we also want to know for sure, whether this then holds for all points in cross-ratio space, either in the ears $(3 \rightarrow 3)$ or in the bag $(2 \leftrightarrow 4)$.

By explicit inspection one finds, that all listed sign options, except case a1) and case $\mathrm{d}^{*}$ ) correspond to null hexagons, which have at least one vertex $x_{j}$, such that the remaining three non-adjacent vertices $x_{j+2}, x_{j-2}$ and $x_{j+3}$ are all inside the light cone of $x_{j}$ or all outside this light cone. This is a necessary condition for treating them with standard configurations, which are deformations of certain two-dimensional set-ups as introduced in [14]. By scanning then all options for these standard configurations it turns out, that indeed all cases beyond the two exceptions can be covered, for details see appendix $\mathrm{C}$.

We show in appendix D, that the exceptional option of case $\mathrm{d}^{*}$ ) cannot be realised by real configurations. However, the other exceptional case a1) is possible. An explicit example is presented in appendix D.

\footnotetext{
${ }^{7}$ The seemingly strange notation in the following table anticipates the later finding, that case $\mathrm{d}^{*}$ ) cannot be realised.
} 
Now we can count the number of inequivalent conformal classes for null hexagons (with numbered vertices) belonging to a given point in cross-ratio space. For points in the ears it is $1 \times 2+6 \times 2+3 \times 1=17$ and for points in the bag $6 \times 1+3 \times 1+6 \times 1=15$. In both cases there is an additional factor 4 due to the still open possibility of overall time reversal and parity transformation.

\section{Special configurations}

For a hexagon in a two-dimensional $\mathbb{R}^{1,1}$ subspace one finds

$$
\text { 2D: } \quad\left(u_{1}, u_{2}, u_{3}\right)=(1,1,1) \text {. }
$$

If it lives in a three-dimensional $\mathbb{R}^{1,2}$ subspace, one has $z= \pm 1$ instead of $-1 \leq z \leq 1$ in the discussion around (20). This means that the corresponding point in cross-ratio space has to be on the surface separating the allowed and not allowed region in fig.1, i.e.

$$
\text { 3D: } \quad 4 u_{1} u_{2} u_{3}-\left(u_{1}+u_{2}+u_{3}-1\right)^{2}=0 .
$$

For the degeneration to a pentagon a certain vertex has to coincide with one of its next neighbours. For generic location of the remaining vertices (1) leads to

$$
\text { pentagons: }\left(u_{1}, u_{2}, u_{3}\right)=(1,0,0) \text { or }(0,1,0) \text { or }(0,0,1) \text {. }
$$

The existence of these three pentagon points in (43) is not a source for different conformal classes for pentagons. They only reflect the freedom one has in choosing the location for the sixth edge, if one wants to extend a pentagon back to a hexagon.

In a collinear limit one of the $x_{j j+2}^{2}$ is zero (without having a pentagon case). Then necessarily one of the cross-ratios is zero, and in addition (20) implies that the sum of the other two cross-ratios is equal to one, i.e.

$$
\text { collinear limits: } u_{j}=0 \text { and } u_{j+1}+u_{j+2}=1, \quad \text { for } j=1,2 \text { or } 3 \text {. }
$$

For hexagons with two crossing edges it has been shown in [15], [16, [9], that

$$
\text { crossing edges: } u_{j}=1 \text { and } u_{j+1}=u_{j+2}, \quad \text { for } j=1,2 \text { or } 3 \text {. }
$$

At this point an amusing side remark is in order. As mentioned in the introduction, in an appendix of [9] an explicit example has been discussed for two hexagon configurations with and without self-crossing, having the same cross-ratios of type (45). It has been used as an argument in favour of the existence of different conformal classes for a given set of cross-ratios. From our discussion in section 2 we now know that there must exist a conformal transformation, which maps the two sets of vertex points to each other. Nevertheless the two hexagons of that example are not conformally equivalent. They use as edges different parts of the null geodesics defined by two adjacent vertices. In the case with no crossing in finite Minkowski space the crossing is just at infinity, in the sense discussed in our appendix B. 
Other interesting limiting cases in cross-ratio space are connected with multi Regge limits [17, 18]. They correspond to a special parametrisation for an approach to the pentagon points (43), either from inside the bag ( $2 \leftrightarrow 4$ scattering) or from ears 1 to 3 (3 $\rightarrow 3$ scattering).

Before closing this section we comment on motions in cross-ratio space (see figs:1 and 2) generated by continuous deformations of hexagons. Transitions between the bag and the ears and among the ears are possible only via sign changes of Mandelstam variables. The signs of the $x_{j j+2}^{2}$ are responsible for the distinction between bag and ears. Besides realisation in passing through the $2 \mathrm{D}$ point $(1,1,1)$, a sign change of one of the $x_{j j+2}^{2}$ can take place only if one crosses one of the pentagon points 8 .

The distinction between the ears is due to the signs of the $x_{j j+3}^{2}$. If one of them changes sign by passing zero, two cross ratios change their sign by passing infinity. To make these transitions more easy on the eyes we depict in fig.2 the allowed crossratios in terms of $v_{j}:=\frac{1}{u_{j}}, j=1,2,3$. Ear 4 of fig 1 is mapped to the central region in $v$-space. Ear 1,2 and 3 appear again as ears extending into the regions with two negative $v$ and one positive $v$. The bag of fig 1 is mapped into part of the region where all $v_{j}>1$.

Now the transitions take place on the pieces of the $v_{1}, v_{2}$ or $v_{3}$ axis where the images of two ears touch each other.
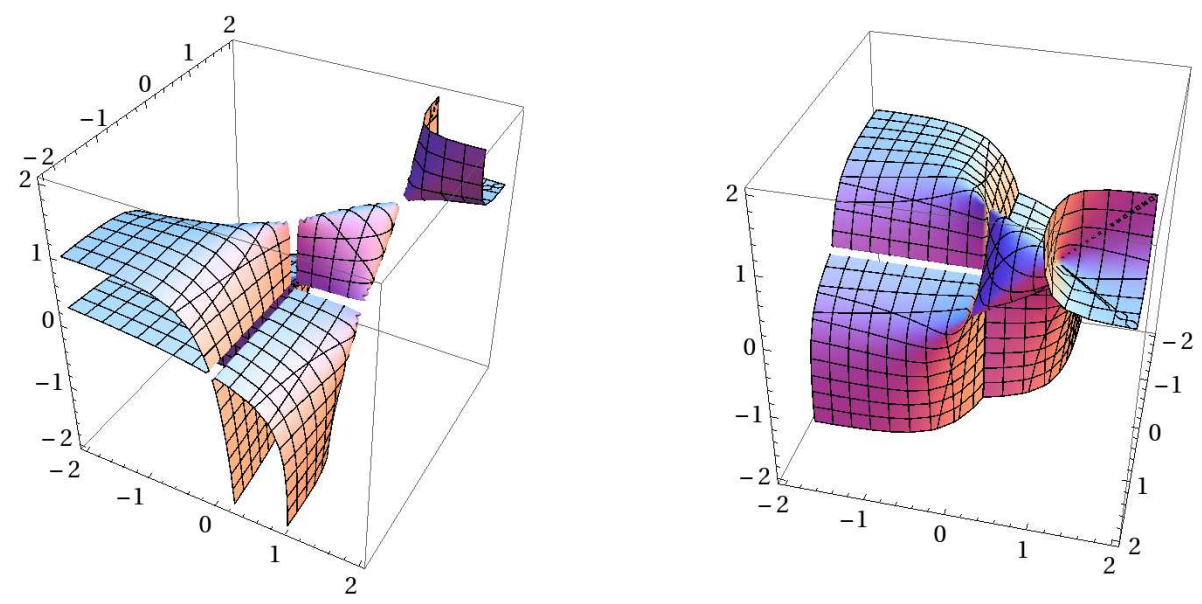

Figure 2: Two views of the allowed region for null hexagons in the $v=1 / u$ space. There is still one more component of the bounding surface not shown in these pictures. It crosses the diagonal at $v_{1}=v_{2}=v_{3}=4$.

\section{Conclusions}

We have given a complete characterisation of the region in cross-ratio space, which corresponds to real null hexagon configurations in $\mathbb{R}^{1,3}$. To each generic point in the

\footnotetext{
${ }^{8}$ They can become zero also by approaching the collinear lines (44), but away from the pentagon points there one is faced with a local maximum/minimum situation.
} 
allowed region corresponds, up to time reversal, parity and up to the cyclic permutation $\left(x_{1}, \ldots, x_{6}\right) \rightarrow\left(x_{4}, \ldots, x_{3}\right)$, just one class of conformally equivalent ordered sets of six points with null separated neighbours.

Using these six points as vertices of null hexagons one has the option to include or exclude hexagons which close via infinity. In the first case one gets then for each class of the ordered 6 -tuples $4 \times 2^{6}$ conformal classes of null hexagons.

Taking into account only null hexagons closed in finite domains of $\mathbb{R}^{1,3}$, one has to exclude special conformal transformations whose critical light cone crosses the hexagons. Then the signs of the Mandelstam variables become invariants. As a result we found 17 conformal classes for generic points in the cross-ratio region corresponding to $3 \rightarrow 3$ scattering and 15 classes in the region corresponding to $2 \leftrightarrow 4$ scattering (by time reversal and parity there is still a factor 4 ).

After our purely geometrical analysis further studies should yield insight into the following issues. Are the remainder functions of Wilson loops for different conformal classes, but the same point in cross ratio space, related by analytic continuation? A special example for such a continuation has been analysed in [9], but it has to be clarified whether analytic continuations connect all classes. Is there some new information in Wilson loops for contours closed only via infinity?

For the investigation of these questions one should also keep in mind, that there exists an alternative conformal compactification of Minkowski space. It differs from that used in section 2 by restricting oneself in the definition of equivalence classes to $\lambda>09$. As a consequence, $\mathbb{R}^{1,3}$ is mapped to half of the modified projective cone. The version used above is obtained back by identification of antipodal points.

After extension to the universal covering, this setting turned out to be appropriate for a causal quantisation of conformally invariant field theories and its relation to the corresponding Euclidean theory, see [12] and refs. therein.

Our option to allow hexagon configurations closing via infinity comes then back as the option to play with edges connecting a certain vertex to the antipode of its neighbour.

Concerning Wilson loops for contours closed only via infinity another issue seems to be of interest for further investigation. It is the relation to the anomaly 10 with respect to inversions studied for Euclidean $\mathcal{N}=4$ super Yang-Mills theory in refs. [21,22]. The Wilson loop for a straight line is equal to one, but that for a circle equal to a nontrivial function of the coupling constant, although a circle touching the origin is mapped to a straight line under inversion at the unit sphere. Since in the Euclidean version only one point of a closed contour passing the origin is mapped to infinity, it has been argued by locality that the relative factor between Wilson loops before and after the inversion is universal for smooth contours [21].

In Minkowski space at least perturbative calculations should be feasible before and after application of a special conformal transformation which opens a closed contour. At the strong coupling end one should be able to handle the situation for instance for

\footnotetext{
${ }^{9}$ For an early parallel discussion of both options see [20.

${ }^{10}$ This anomaly has to be distinguished from the anomaly with respect to infinitesimal conformal transformations [5], which via differential equations governs for instance the UV divergencies due to cusps of the contour and also parts of the renormalised Wilson loops.
} 
null tetragons where the corresponding AdS string surface is known explicitely 2].

The conformal geometry of null polygons as analysed in the present paper could be also usefully beyond $\mathcal{N}=4$ super Yang-Mills theory, for instance in QCD, after one succeeds in separating conformal invariant pieces of the corresponding Wilson loops.

\section{Acknowledgement}

H.D. thanks G. Jorjadze and S. Wuttke for helpful discussions and E. Radatz for collaboration in an early stage. The work is supported in part by DFG via SFB 647 and by VolkswagenStiftung via grant I/84600. 


\section{Appendix A}

We are interested in the explicit form of the $S O(2,4)$ transformations acting in $\mathbb{R}^{2,4}$, which correspond to finite translations, Lorentz transformations, dilatations and special conformal transformations. For some related discussion on the level of infinitesimal transformations see e.g. [19]. If we map from Minkowski space to $\mathbb{R} P_{2,4}^{5}$ with (4), perform $\Lambda \in S O(2,4)$ in $\mathbb{R}^{2,4}$ and then go back to Minkowski via (5) we get

$$
x^{\prime \mu}=\frac{\Lambda_{\nu}^{\mu} x^{\nu}+\frac{1}{2}\left(\Lambda_{0^{\prime}}^{\mu}+\Lambda_{4}^{\mu}\right)-\frac{1}{2}\left(\Lambda_{0^{\prime}}^{\mu}-\Lambda_{4}^{\mu}\right) x^{2}}{\left(\Lambda_{\nu}^{0^{\prime}}+\Lambda^{4}{ }_{\nu}\right) x^{\nu}+\frac{1}{2}\left(\Lambda_{0^{\prime}}^{0^{\prime}}+\Lambda_{0^{\prime}}^{4}+\Lambda_{4}^{0^{\prime}}+\Lambda_{4}^{4}\right)-\frac{1}{2}\left(\Lambda_{0^{\prime}}^{0^{\prime}}+\Lambda_{0^{\prime}}^{4}-\Lambda_{4}^{0^{\prime}}-\Lambda_{4}^{4}\right) x^{2}} .
$$

To realise a translation $x^{\prime \mu}=x^{\mu}+a^{\mu}$ one has to choose $\Lambda_{\nu}^{\mu}=\delta_{\nu}^{\mu}, \quad\left(\Lambda_{0^{\prime}}^{\mu}+\Lambda_{4}^{\mu}\right)=$ $2 a^{\mu}, \quad\left(\Lambda_{0^{\prime}}^{0^{\prime}}+\Lambda_{0^{\prime}}^{4}+\Lambda_{4}^{0^{\prime}}+\Lambda_{4}^{4}\right)=2$ and the remaining coefficients of $x^{\nu}$ or $x^{2}$ in (46) equal to zero. Together with the $S O(2,4)$ condition $\eta^{K L}=\Lambda_{A}^{K} \eta^{A B} \Lambda_{B}^{L}$ this fixes $\Lambda$ up to the freedom $\Lambda \rightarrow-\Lambda 11$

$$
\underline{\text { translation: }} \Lambda_{N}^{M}=\left(\begin{array}{ccc}
1 & a^{\mu} & a^{\mu} \\
-a_{\nu} & 1-\frac{a^{2}}{2} & -\frac{a^{2}}{2} \\
a_{\nu} & \frac{a^{2}}{2} & 1+\frac{a^{2}}{2}
\end{array}\right) \text {. }
$$

In a similar manner we get $x^{\prime \mu}=\frac{x^{\mu}+c^{\mu} x^{2}}{1+2 c x+c^{2} x^{2}}$ via

$$
\underline{\text { special conformal: }} \Lambda_{N}^{M}=\left(\begin{array}{ccc}
\mathbb{1} & -c^{\mu} & c^{\mu} \\
c_{\nu} & 1-\frac{c^{2}}{2} & \frac{c^{2}}{2} \\
c_{\nu} & -\frac{c^{2}}{2} & 1+\frac{c^{2}}{2}
\end{array}\right) \text {. }
$$

Lorentz transformations in Minkowski space correspond to

$$
\text { Lorentz: } \Lambda_{N}^{M}=\left(\begin{array}{ccc}
\Lambda^{\mu}{ }_{\nu} & 0 & 0 \\
0 & 1 & 0 \\
0 & 0 & 1
\end{array}\right), \quad \Lambda_{\nu}^{\mu} \in S O(1,3)
$$

And finally, dilatations $x^{\prime \mu}=e^{-\rho} x^{\mu}$ have as partner

$$
\underline{\text { dilatation: }} \Lambda_{N}^{M}=\left(\begin{array}{ccc}
\mathbb{1} & 0 & 0 \\
0 & \cosh \rho & \sinh \rho \\
0 & \sinh \rho & \cosh \rho
\end{array}\right) \text {. }
$$

\section{Appendix B}

Here we collect some properties of the images of Minkowski space null geodesics in $\mathbb{R} P_{2,4}^{5}$. Null geodesic have the form $x+t n$ with $x, n \in \mathbb{R}^{1,3}, n^{2}=0, n^{0}= \pm 1, \quad t \in \mathbb{R}$.

Via (41) the corresponding image is given by $W(t)=\lambda\left(n+\frac{x}{t}, \frac{1-x^{2}}{2 t}-x n, \frac{1+x^{2}}{2 t}+x n\right)$.

For $t \rightarrow \pm \infty$ we get the same point, it is

$$
\begin{aligned}
& W(\infty)=\lambda\left(\frac{n^{\mu}}{x n},-1,1\right) \text { for } x n \neq 0, \\
& W(\infty)=\lambda\left(n^{\mu}, 0,0\right) \text { for } x n=0 .
\end{aligned}
$$

\footnotetext{
${ }^{11}$ For the capital indices $M, N$ etc. we take for convenience the ordering $0,1,2,3,0^{\prime}, 4$.
} 
Thus null geodesics on conformally compactified Minkowski space 12 have the topology of a circle. For two null geodesics, crossing at $x$ with different directions $n_{1}, n_{2}$, the corresponding points at infinity are different. Two different null geodesics do either not intersect or intersect at only one point.

If one asks for the condition on $x_{1}, n_{1}$ and $x_{2}, n_{2}$ for crossing at infinity one gets

$$
n_{2}^{\mu}= \pm n_{1}^{\mu}, \quad x_{1} n_{1}=x_{2} n_{1}
$$

\section{Appendix C}

In [14] conformal transformations where used to map a certain class to the standard configuration $(\Lambda \rightarrow+\infty)$

$$
\begin{gathered}
x_{3}=(0,0,0), x_{2}=\left(-\frac{1+\vec{p}^{2}}{2}-\frac{1-\vec{p}^{2}}{2}, \vec{p}\right), x_{4}=\left(-\frac{1+\vec{q}^{2}}{2}, \frac{1-\vec{q}^{2}}{2}, \vec{q}\right), \\
x_{1}=x_{2}+(\Lambda / 2,-\Lambda / 2,0), x_{5}=x_{4}+(\Lambda / 2, \Lambda / 2,0), x_{6}=(\Lambda, 0,0)+\ldots .
\end{gathered}
$$

Note the different role of the two-dimensional transversal vectors $\vec{p}$ and $\vec{q}$ compared to our treatment in section 2. Here they are used to move the points staying finite into the transversal directions, there they move transversally the points, which are put into conformal infinity.

The resulting formulas for the cross-ratios have the same form as (16)).

In the following we need 8 more configurations of a similar nature as that in (53). We call them all standard configurations and show the Penrose diagrams of their projection on $\mathbb{R}^{1,1}$ (for $\vec{p}=\vec{q}=0$ ) in fig, 3 . The assignments of the vertex number to the vertices in fig. 3 is fixed by the sequence of plus/minus in table (34). Only in case a) (corresponding to standard configuration 1)) we can make use of cyclically permuted vertex assignments.

It is straightforward to check that standard configurations 1,3,4,5,6 and 9 yield cross-ratio formulas looking, up to a possible permutation of the $u_{j}$, as (16) and standard configurations 2,7 and 8 yield the form (18). Varying $\vec{p}$ and $\vec{q}$, each of these 9 standard configurations covers either the complete ear or bag region in cross-ratio space. To check which of the classes of tables (35)-(40) are realised, we have to consider the sign pattern for the Mandelstam variables $\left\{x_{j j+2}^{2}\right\}$ and $\left\{x_{j j+3}^{2}\right\}$ in these standard configurations. In each of these cases the sign of all $\left\{x_{j j+2}^{2}\right\}$ is fixed. Beyond this also the sign of that Mandelstam variable out of $\left\{x_{j j+3}^{2}\right\}$, which is related to the point in the origin, is fixed and can be read off from fig 3 . The sign of the other two is also fixed for the bag configurations, but can take independently the values \pm for ear configurations. This yields the following table

\footnotetext{
${ }^{12}$ Its topology is $S^{1} \times S^{3}$, see e.g. [13].
} 


\begin{tabular}{|c|l||c|l|}
\hline stand. config. & conf. classes & stand. config. & conf. classes \\
\hline \hline $\mathbf{1}, x_{3}$ in origin & $\mathrm{a} 2, \mathrm{a} 4, \mathrm{a} 5, \mathrm{a} 6$ & $\mathbf{5}$ & $\mathrm{c} 2, \mathrm{c} 3, \mathrm{c} 5, \mathrm{c} 7$ \\
\hline $\mathbf{1}, x_{2}$ in origin & $\mathrm{a} 2, \mathrm{a} 3, \mathrm{a} 5, \mathrm{a} 7$ & $\mathbf{6}$ & $\mathrm{c} 2, \mathrm{c} 3, \mathrm{c} 4, \mathrm{c} 8$ \\
\hline $\mathbf{1}, x_{1}$ in origin & $\mathrm{a} 2, \mathrm{a} 3, \mathrm{a} 4, \mathrm{a} 8$ & $\mathbf{7}$ & $\mathrm{e}$ \\
\hline $\mathbf{2}$ & $\mathrm{b}$ & $\mathbf{8}$ & $\mathrm{d}$ \\
\hline $\mathbf{3}$ & $\mathrm{c} 2, \mathrm{c} 4, \mathrm{c} 5, \mathrm{c} 6$ & $\mathbf{9}$ & $\mathrm{f} 1, \mathrm{f} 2, \mathrm{f} 3, \mathrm{f} 4$ \\
\hline $\mathbf{4}$ & $\mathrm{c} 1, \mathrm{c} 3, \mathrm{c} 7, \mathrm{c} 8$ & & \\
\hline
\end{tabular}

Now we see that all conformal classes of (35)-(40), except a1) and $\left.\mathrm{d}^{*}\right)$, can be realised by standard configurations sketched in fig. 3 .

(1)

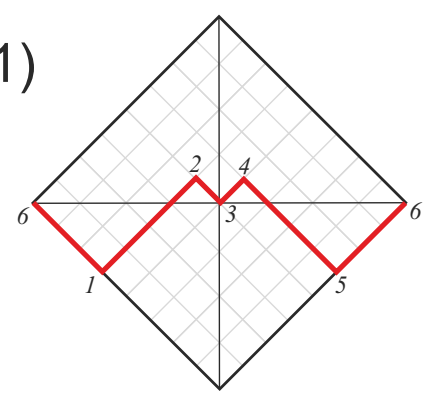

(4)

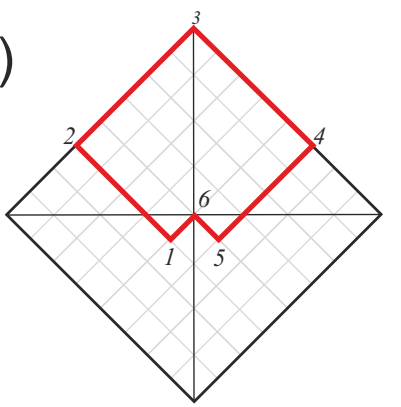

(7)

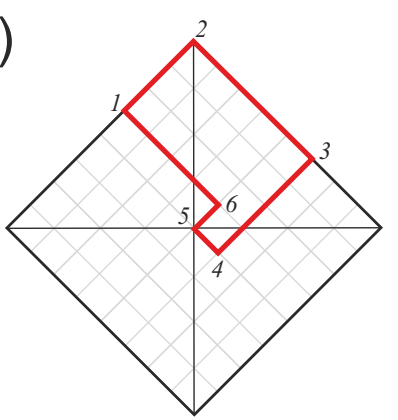

(2)

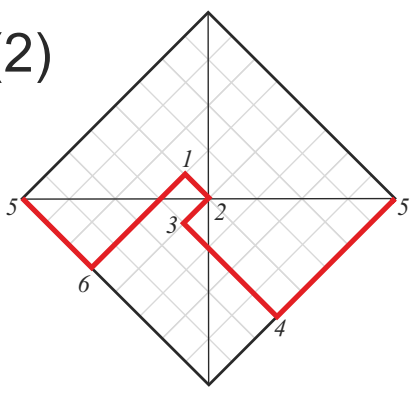

(5)

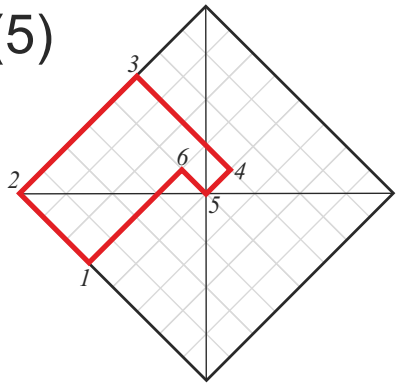

(8)

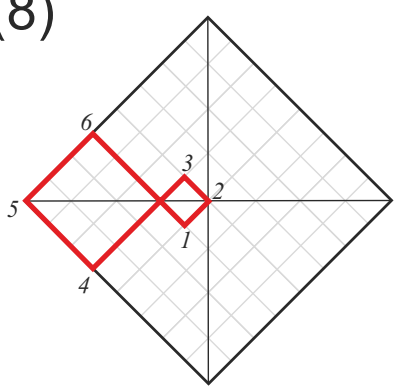

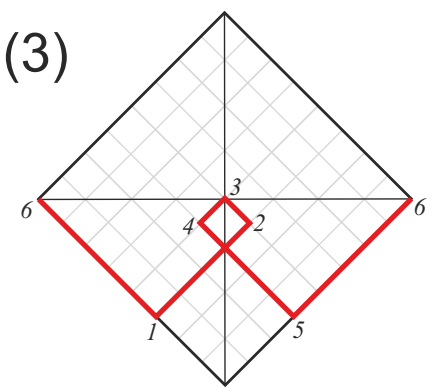

(3)

(6)

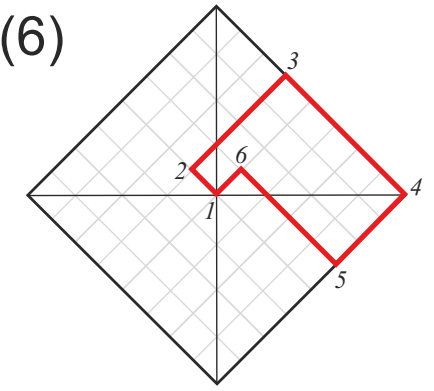

(9)

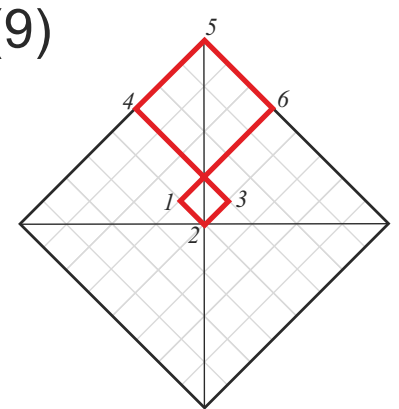

Figure 3: Penrose diagrams of $2 D$ projection of standard configurations at $\vec{p}=\vec{q}=0$. The case in [14] and presented in eq. (53) corresponds to picture (4) after a suitable cyclic permutation of the vertices.

\section{Appendix D}

We first prove that generically there do not exist real configurations of type $\mathrm{d}^{*}$ ). In general, a generic null hexagon configuration can be characterised in the following 
way. Consider a hexagon in $\mathbb{R}^{3}$, spanned by the edges $\vec{p}_{1}, \ldots \vec{p}_{6}$. With $p_{j}^{0}= \pm\left|\vec{p}_{j}\right|$ one can see it as the space projection of a cusped null line in $\mathbb{R}^{1,3}$. This null line becomes a (closed) hexagon iff the sums of the three-dimensional lengths of edges, corresponding to the plus/minus choice for the time component, are equal to each other.

Applied to the type $\mathrm{d}^{*}$ ) this imposes the condition

$$
\left|\vec{p}_{3}\right|+\left|\vec{p}_{6}\right|=\left|\vec{p}_{1}\right|+\left|\vec{p}_{2}\right|+\left|\vec{p}_{4}\right|+\left|\vec{p}_{5}\right| .
$$

To realise the $\mathrm{d}^{*}$ ) set-up, all the $x_{j j+3}^{2}$ would have to be positive. This would imply, that in a suitable Lorentz frame the space components of e.g. vertices 1 and 4 coincide: $\vec{x}_{1}=\vec{x}_{4}$, i.e.

$$
\vec{p}_{1}+\vec{p}_{2}+\vec{p}_{3}=0 \quad \text { and } \quad \vec{p}_{4}+\vec{p}_{5}+\vec{p}_{6}=0 .
$$

By the triangle inequality one has $\left|\vec{p}_{3}\right| \leq\left|\vec{p}_{1}\right|+\left|\vec{p}_{2}\right|$ and $\left|\vec{p}_{6}\right| \leq\left|\vec{p}_{4}\right|+\left|\vec{p}_{5}\right|$. This is in conflict with (54) (of course except in degenerated cases where both triangles behind (55) collapse to a line).

The just used three-dimensional point of view is also helpful to find explicit examples for real configurations of type a1). Since also here $x_{14}^{2}$ is positive, in a suitable Lorentz frame the 3-dimensional hexagon becomes a pair of triangles, but now, instead of (55), with the condition

$$
\left|\vec{p}_{1}\right|+\left|\vec{p}_{3}\right|+\left|\vec{p}_{5}\right|=\left|\vec{p}_{2}\right|+\left|\vec{p}_{4}\right|+\left|\vec{p}_{6}\right| .
$$

This imposes no obstruction by the triangle equations, and just putting the two triangles on top of each other gives an explicit example for a type a1) configuration in a $\mathbb{R}^{1,2}$ subspace

$$
\begin{aligned}
& x_{1}=(0,0,0), \quad x_{2}=(1,1,0), \quad x_{3}=\left(0, \frac{1}{2}, \frac{\sqrt{3}}{2}\right), \\
& x_{4}=(1,0,0), \quad x_{5}=(0,1,0), \quad x_{6}=\left(1, \frac{1}{2}, \frac{\sqrt{3}}{2}\right) .
\end{aligned}
$$

It is depicted in fig, 4, The related cross-ratios are $u_{1}=u_{2}=u_{3}=1$. By rotating the two triangles out of the coincidence position, rotating one of them into the third space direction or playing with the lengths of the triangle sides, one for sure has enough freedom to reach all points in ear 4 by deformations of the special configuration (57). 


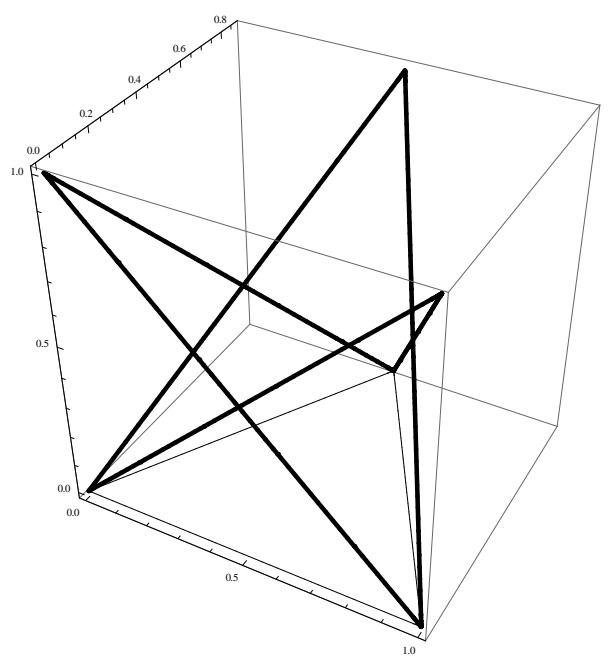

Figure 4: Example for a configuration in class a1). The timelike coordinate points upwards. With thin lines the projection to the spacelike $\left(x^{1}, x^{2}\right)$-plane is indicated.

\section{Appendix E}

Here we show that, as soon as the sign of $\left(x_{i}-x_{j}\right)^{2}$ changes under a conformal transformation, the corresponding image of each continuous contour connecting $x_{i}$ and $x_{j}$ passes infinity. Let us denote by $W_{i}$ and $W_{j}$ the related points on the cone in $\mathbb{R}^{2,4}$. Then $\operatorname{sign}\left(\left(x_{1}^{\prime}-x_{2}^{\prime}\right)^{2}\right)=-\operatorname{sign}\left(\left(x_{1}-x_{2}\right)^{2}\right)$ via (6) implies $(\Lambda \in S O(2,4))$

$$
\begin{aligned}
& \operatorname{sign}\left(\left(\Lambda W_{1}\right)^{0^{\prime}}+\left(\Lambda W_{1}\right)^{4}\right)= \pm \operatorname{sign}\left(W_{1}^{0^{\prime}}+W_{1}^{4}\right), \\
& \operatorname{sign}\left(\left(\Lambda W_{2}\right)^{0^{\prime}}+\left(\Lambda W_{2}\right)^{4}\right)=\mp \operatorname{sign}\left(W_{2}^{0^{\prime}}+W_{2}^{4}\right) .
\end{aligned}
$$

Let us now consider the function

$$
f_{\Lambda}(W):=\frac{(\Lambda W)^{0^{\prime}}+(\Lambda W)^{4}}{W^{0^{\prime}}+W^{4}}
$$

and evaluate it along a continuous contour from $W_{1}$ to $W_{2}$, whose image in $\mathbb{R}^{1,3}$ stays in a finite region. Due to (58) it has opposite sign on both ends. Hence one necessarily finds zeros along the contour. These zeros of $(\Lambda W)^{0^{\prime}}+(\Lambda W)^{4}$ correspond to passing conformal infinity of $\mathbb{R}^{1,3}$. 


\section{References}

[1] G. P. Korchemsky and A. V. Radyushkin, Nucl. Phys. B 283 (1987) 342.

[2] L. F. Alday and J. M. Maldacena, JHEP 0706 (2007) 064 arXiv:0705.0303 [hepth]]. • L. F. Alday and J. Maldacena, JHEP 0711 (2007) 068 arXiv:0710.1060 [hep-th]].

[3] J. M. Drummond, J. Henn, G. P. Korchemsky and E. Sokatchev, Nucl. Phys. B 795 (2008) 52 [arXiv:0709.2368 [hep-th]].

[4] J. M. Drummond, J. Henn, G. P. Korchemsky and E. Sokatchev, Nucl. Phys. B 815 (2009) 142 arXiv:0803.1466 [hep-th]].

[5] J. M. Drummond, J. Henn, G. P. Korchemsky and E. Sokatchev, Nucl. Phys. B 826 (2010) 337 arXiv:0712.1223 [hep-th]].

[6] C. Anastasiou, Z. Bern, L. J. Dixon and D. A. Kosower, Phys. Rev. Lett. 91 (2003) 251602 [arXiv:hep-th/0309040]. • Z. Bern, L. J. Dixon and V. A. Smirnov, Phys. Rev. D 72 (2005) 085001 [arXiv:hep-th/0505205].

[7] C. Anastasiou, A. Brandhuber, P. Heslop, V. V. Khoze, B. Spence and G. Travaglini, JHEP 0905 (2009) 115 [arXiv:0902.2245 [hep-th]].

[8] D. Gaiotto, J. Maldacena, A. Sever and P. Vieira, JHEP 1112 (2011) 011 arXiv:1102.0062 [hep-th]].

[9] H. Dorn and S. Wuttke, JHEP 1204 (2012) 023 [arXiv:1111.6815 [hep-th]].

[10] P. A. M. Dirac, Annals Math. 37 (1936) 429.

[11] H. A. Kastrup, Phys. Rev. 150 (1966) 1183. • G. Mack and A. Salam, Annals Phys. 53 (1969) 174. • G. Mack and I. T. Todorov, Phys. Rev. D 8 (1973) 1764 [Phys. Rev. D 6 (1973) 1764]. • W. Siegel, "Embedding versus 6D twistors," arXiv:1204.5679 [hep-th].

[12] M. Lüscher and G. Mack, Commun. Math. Phys. 41 (1975) 203.

[13] G. W. Gibbons and A. R. Steif, Phys. Lett. B 346 (1995) 255 hep-ph/9412210.

[14] L. F. Alday, D. Gaiotto and J. Maldacena, JHEP 1109 (2011) 032. [arXiv:0911.4708 [hep-th]].

[15] G. Georgiou, JHEP 0909 (2009) 021 [arXiv:0904.4675 [hep-th]].

[16] H. Dorn and S. Wuttke, JHEP 1105 (2011) 114 [arXiv:1104.2469 [hep-th]].

[17] L. N. Lipatov and A. Prygarin, Phys. Rev. D 83 (2011) 125001 arXiv:1011.2673 [hep-th]]. 
[18] J. Bartels, L. N. Lipatov and A. Prygarin, Phys. Lett. B 705 (2011) 507 arXiv:1012.3178 [hep-th]].

[19] C. Codirla and H. Osborn, Annals Phys. 260 (1997) 91 hep-th/9701064.

[20] L. Castell, Nucl. Phys. B 13 (1969) 231.

[21] N. Drukker, D. J. Gross, J. Math. Phys. 42 (2001) 2896 [hep-th/0010274].

[22] G. W. Semenoff, K. Zarembo, Nucl. Phys. Proc. Suppl. 108 (2002) 106 hep-th/0202156. 